This item was submitted to Loughborough's Research Repository by the author.

Items in Figshare are protected by copyright, with all rights reserved, unless otherwise indicated.

\title{
Evaluation of genipin-crosslinked chitosan hydrogels as a potential carrier for silver sulfadiazine nanocrystals
}

PLEASE CITE THE PUBLISHED VERSION

http://dx.doi.org/10.1016/j.colsurfb.2016.06.016

PUBLISHER

(C) Elsevier

VERSION

AM (Accepted Manuscript)

\section{PUBLISHER STATEMENT}

This work is made available according to the conditions of the Creative Commons Attribution-NonCommercialNoDerivatives 4.0 International (CC BY-NC-ND 4.0) licence. Full details of this licence are available at: https://creativecommons.org/licenses/by-nc-nd/4.0/

\section{LICENCE}

CC BY-NC-ND 4.0

\section{REPOSITORY RECORD}

Gao, Lei, Hui Gan, Zhiyun Meng, Ruolan Gu, Zhuona Wu, Xiaoxia Zhu, Wenzhong Sun, et al.. 2016. "Evaluation of Genipin-crosslinked Chitosan Hydrogels as a Potential Carrier for Silver Sulfadiazine Nanocrystals". figshare. https://hdl.handle.net/2134/22569. 


\section{Accepted Manuscript}

Title: Evaluation of genipin-crosslinked chitosan hydrogels as a potential carrier for silver sulfadiazine nanocrystals

Author: Lei Gao Hui Gan Zhiyun Meng Ruolan Gu Zhuona Wu Xiaoxia Zhu Wenzhong Sun Jian Li Ying Zheng Tao Sun Guifang Dou

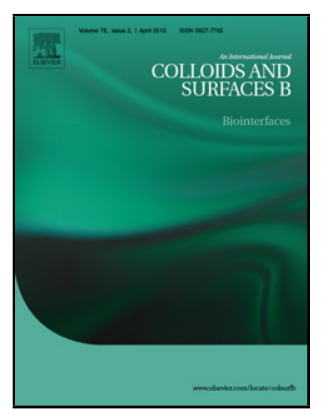

PII: S0927-7765(16)30446-5

DOI: http://dx.doi.org/doi:10.1016/j.colsurfb.2016.06.016

Reference: COLSUB 7959

To appear in: $\quad$ Colloids and Surfaces B: Biointerfaces

Received date: $\quad 20-2-2016$

Revised date: 22-5-2016

Accepted date: $\quad$ 9-6-2016

Please cite this article as: Lei Gao, Hui Gan, Zhiyun Meng, Ruolan Gu, Zhuona Wu, Xiaoxia Zhu, Wenzhong Sun, Jian Li, Ying Zheng, Tao Sun, Guifang Dou, Evaluation of genipin-crosslinked chitosan hydrogels as a potential carrier for silver sulfadiazine nanocrystals, Colloids and Surfaces B: Biointerfaces http://dx.doi.org/10.1016/j.colsurfb.2016.06.016

This is a PDF file of an unedited manuscript that has been accepted for publication. As a service to our customers we are providing this early version of the manuscript. The manuscript will undergo copyediting, typesetting, and review of the resulting proof before it is published in its final form. Please note that during the production process errors may be discovered which could affect the content, and all legal disclaimers that apply to the journal pertain. 


\section{Evaluation of genipin-crosslinked chitosan hydrogels as a potential carrier for silver sulfadiazine nanocrystals}

Lei Gao ${ }^{\mathrm{a}, \mathrm{b}}$, Hui Gan ${ }^{\mathrm{a}}$, Zhiyun Meng ${ }^{\mathrm{a}}$, Ruolan $\mathrm{Gu}^{\mathrm{a}}$, Zhuona Wu ${ }^{\mathrm{a}}$, Xiaoxia Zhu ${ }^{\text {a }}$, Wenzhong Sun ${ }^{\text {a }}$, Jian $\mathrm{Li}^{\text {a }}$, Ying Zheng ${ }^{\text {a }}$, Tao Sun ${ }^{\mathrm{c}}$, Guifang Dou ${ }^{\mathrm{a},{ }^{*}}$

${ }^{\text {a }}$ Laboratory of Hematological Pharmacology, Beijing Institute of Transfusion Medicine, China

${ }^{\mathrm{b}}$ Center for Drug Evaluation, State Food and Drug Administration, Beijing, 100038, China

c Centre for Biological Engineering, Department of Chemical Engineering, Loughborough University, Loughborough Leicestershire, LE11 3TU

\section{Corresponding author: Guifang Dou}

Affiliation: Laboratory of Hematological Pharmacology, Beijing Institute of Transfusion Medicine, China

Address: No.27, Taiping Road, Haidian District, Beijing 100850, China

Email: douguifang@vip.163.com

Tel and Fax: 86-10-66931993

\section{Statistical summary}

The whole paper contains about 5900 words, 4 figures and 4 tables.

\section{Conflict of interest}

No 


\section{Graphical Abstract}

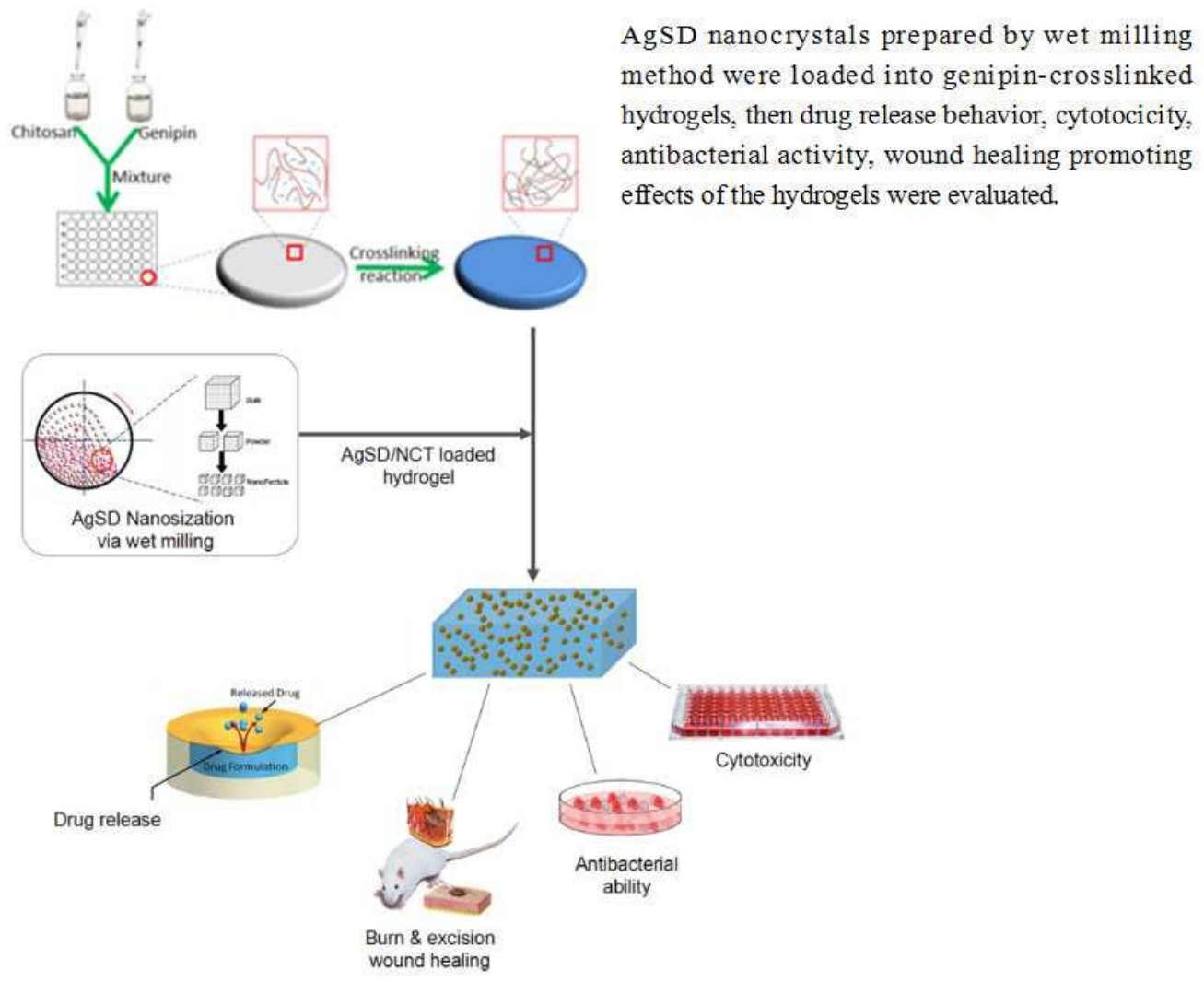

Abbreviations: AgSD, Silver Sulfadiazine;

NCT, Nanocrystal 


\section{Highlights}

1. AgSD nanocrystals improved the dissolution behavior but aggravate the cytotoxicty.

2. Encapsulation of AgSD nanocrystals in chitosan hydrogels reduced their cytotoxicity.

3. Encapsulation of AgSD nanocrystals in hydrogels facilitated sustained release.

4. Encapsulation of AgSD nanocrystals in hydrogels remained high antibacterial activity.

5. Hydrogels containing AgSD nanocrystals showed the best in vivo healing effects. 


\begin{abstract}
:
In the present study genipin crosslinked chitosan (CHI) hydrogels, which had been constructed and reported in our previous studies (Lei Gao, et al. Colloids Surf. B Biointerfaces. 2014, 117: 398), were further evaluated for their advantage as a carrier for silver sulfadiazine (AgSD) nanocrystal systems. Firstly, AgSD nanocrystals with a mean particle size of $289 \mathrm{~nm}$ were prepared by wet milling method and encapsulated into genipin crosslinked CHI hydrogels. AgSD nanocrystals displayed a uniform distribution and very good physical stability in the hydrogel network. Swelling-dependent release pattern was found for AgSD nanocrystals from hydrogels and the release profile could be well fitted with Peppas equation. When AgSD nanocrystals were encapsulated in hydrogels their fibroblast cytotoxicity decreased markedly, and their antibacterial effects against Staphylococcus aureus, Escherichia coli and Pseudomonas aeruginosa were still comparable to unencapsulated AgSD nanocrystals. In vivo evaluation in excision and burn cutaneous wound models in mice showed that AgSD nanocrystal hydrogels markedly decreased the expression of inflammatory cytokine IL-6, but increased the levels of growth factors VEGF-A and TGF- $\beta 1$. Histopathologically, the wounds treated by hydrogels containing AgSD nanocrystals showed the best healing state compared with commercial AgSD cream, hydrogels containing AgSD bulk powders and blank hydrogels. The wounds treated by AgSD nanocrystal hydrogels were dominated by marked fibroblast proliferation, new blood vessels and thick regenerated epithelial layer. Sirius Red staining assay indicated that AgSD nanocrystal hydrogels resulted in more collagen deposition characterized by a large proportion of type I fibers. Our study suggested that genipin-crosslinked $\mathrm{CHI}$ hydrogel was a potential carrier for local antibacterial nanomedicines.
\end{abstract}


Keywords: Silver Sulfadiazine; chitosan; hydrogel; antibacterial; wound healing 


\section{Introduction}

It has been proved that skin wounds are susceptible to infections caused by gram-positive bacteria like Staphylococcus aureus, and gram-negative bacteria like Escherichia coli, Pseudomonas aeruginosa [1]. Infection of wound surfaces often causes delayed healing or nonhealing of the wound, which results in mortality [2]. It was reported that infection was responsible for $75 \%$ of all deaths in patients with burns exceeding $40 \%$ of the total body surface area [3].

Usually skin wounds are rich in exudation and proteins, and consist of avascular necrotic tissues, which limit the effective permeation of systemically administered antibiotics $[4,5]$. Subsequently, topical antibacterial preparations are more suitable in these cases. AgSD, a combination of silver and sulfadiazine, is considered as first-line drug for topical wound management due to its biocidal activities toward a wide spectrum of microorganisms and its wound convergence promoting effects [6]. AgSD has dual antibacterial effects. The free silver can react with both sulfhydryl groups of bacterial enzymes and DNA, and sulfadiazine can stop the synthesis of DNA by interrupting the production of folate [7]. However, the antibacterial effect of AgSD is badly limited by its poor aqueous solubility [8]. Moreover, AgSD has been shown to be cytotoxic in vitro toward fibroblasts and keratinocytes and consequently to retard wound healing in vivo [9]. These two issues limit topical therapeutic effects of AgSD to a large extend. 
Works by us and other groups had proved that nanocrystal technology was an ideal approach to handle the poor solubility problem [10-12]. It is anticipated that the poor solubility of AgSD could be markedly improved by nanocrystal technology. Then the enhanced solubility as well as the increased interaction between drugs and bacteria caused by the huge surface area can markedly improve their antibacterial effects [13]. On the other hand, however, markedly increased surface area after nanosization may also aggravate the cytotoxicity of $\mathrm{AgSD}$, which would retard wound healing. In addition, physical stability of AgSD nanocrystals would be another problem like other antibacterial nanomedicines (such as nano silver, nano $\mathrm{ZnO}$ ) [14, 15]. Nanocrystals will tend to aggregate to reduce the huge surface energy caused by the large interface between the solid and the medium [16]. Therefore, a suitable carrier might be necessary for the application of AgSD nanosystems on the wound.

Hydrogels have been considered as excellent wound dressings due to the water-saturated networks which mimic the three-dimensional extracellular matrix (ECM) and allow for efficient transport of oxygen, nutrients and metabolic products [17]. Several reviews had summarized and analyzed the merits of polymer hydrogels applied for wound care [18-21]. In our previous work CHI hydrogels were prepared by using natural genipin as crosslinking agent. Crosslinking with genipin showed no influence on the cytocompatibility of $\mathrm{CHI}$ and could effectively adjust the stiffness of $\mathrm{CHI}$ hydrogels which then regulate fibroblast attachment and proliferation [22]. In the present research, the genipin crosslinked CHI hydrogels were further studied for the advantage as a potential carrier dressing for AgSD nanocrystals. 


\section{Materials and Methods}

\subsection{Materials}

Silver sulfadiazine was purchased from Dongbei Pharmacy Company; Thiazoyl blue tetrazolium bromide (MTT), trypsin and ethylenediamine tetraacetic acid (EDTA) were purchased from Sigma Company; CHI (molecular weight: 300-450 kDa; viscosity: 500-800 $\mathrm{mPa} \cdot \mathrm{s}$; degree of deacetylation: $85-95 \%$ ), phosphoric acid, ammonia solution, formaldehyde, dimethyl sulfoxide (DMSO) and ethanol were purchased from Sinopharm Chemical Reagent Co., Ltd. Genipin was purchased from Linchuan Zhixin Biotechnology Company (Fuzhou, China); Acetonitrile (Fisher Company) was of HPLC grade, other reagents were of analytical grade. Ultrapurified water was obtained from a Milli-Q ultrapurification system (Milli-Q ${ }^{\circledR}$ Integral, USA).

\subsection{Wet milling}

AgSD nanocrystal (AgSD/NCT) suspensions were prepared using a wet-milling technique. $0.2 \mathrm{~g}$ CHI was dissolved in $100 \mathrm{ml}$ water. $10 \mathrm{~g}$ of drug powder was dispersed in the $\mathrm{CHI}$ solution. The obtained suspensions were poured into the holder of a nano milling system (Elektromotorenwerk brienz AG) containing $50 \mathrm{~g}$ of milling beads (zirconium oxide, diameter $1 \mathrm{~mm}$ ). The grinding was performed at $1500 \mathrm{rpm}$ 
for $20 \mathrm{~min}$ and $2500 \mathrm{rpm}$ for $20 \mathrm{~min}$, and the holder was cooled at $0^{\circ} \mathrm{C}$ by circulating a refrigerant. Particle size reduction was monitored with a Malvern Zetasizer 3000HS (Malvern Instrument, Malvern, UK) by taking samples at different time intervals during the process. After milling, the nanosuspensions were separated from the grinding beads.

\subsection{Characterization of nanosuspensions}

\subsubsection{Particle size and morphology}

The particle size and polydispersity indexes (PI) of AgSD/NCT suspensions were measured with Malvern Zetasizer (3000SH, Malvern Instruments Ltd., UK). The morphology of nanoparticles was observed using transmission electron microscope (TEM, JEM-1200EX, Japan). One drop of nanosuspension was placed on a copper grid and stained with $2 \%$ phosphotungstic acid for $2 \mathrm{~min}$. The grid was allowed to dry at room temperature and was examined with the electron microscope. All measurements were made in triplicate.

\subsubsection{Dissolution}

Dissolution behavior of AgSD/NCT was studied by using a rotary shaker (HZS-HA, China). $200 \mathrm{~mL}$ phosphate buffer solution (PBS, $\mathrm{pH}=7.4$ ) at $37^{\circ} \mathrm{C}$ was used as dissolution media. Samples containing equivalent of AgSD (10mg) were dispersed into dissolution vessel and shaken at $100 \mathrm{rpm}$. At predetermined intervals, $100 \mu \mathrm{L}$ of 
medium was withdrawn and centrifuged at $14000 \mathrm{rpm}$ for $15 \mathrm{~min}$ at $4^{\circ} \mathrm{C}(3 \mathrm{~K} 18$ centrifuge, Sigma, Germany). Equal blank medium was compensated immediately following each sampling. AgSD content was measured after adequate dilution with mobile phase using an Agilent 1290 ultrahigh-pressure liquid chromatograph (UHPLC) equipped with a quaternary pump, an auto sampler and DAD UV detector. The detection wavelength was set at $260.0 \mathrm{~nm}$. Chromatographic separation was carried out with an Agilent Eclipse Plus C18 Column $(3.5 \mu \mathrm{m}$ particle size, $2.1 \times 50 \mathrm{~mm})$ with mobile phase of $0.1 \%$ phosphoric acid/ acetonitrile $(94: 6, \mathrm{v} / \mathrm{v})$ at $30^{\circ} \mathrm{C}$. The flow rate was $0.30 \mathrm{~mL} / \mathrm{min}$. Dissolution profiles of $\mathrm{AgSD}$ bulk power (AgSD/Bulk) suspensions were also measured under the same conditions.

\subsection{Hydrogel formation}

The AgSD/NCT suspension prepared with $\mathrm{CHI}$ as stabilizer was diluted using $\mathrm{CHI}$ solution to obtain a final concentration of $0.1 \% \mathrm{AgSD}$ and $2 \% \mathrm{CHI}(\mathrm{w} / \mathrm{v})$. To obtain hydrogels of different crosslinking levels, different amount of genipin $(1.65 \mathrm{mM}$, $2.75 \mathrm{mM}, 3.30 \mathrm{mM}$ and $4.40 \mathrm{mM}$, respectively) were added into the resultant suspension followed by vigorous stirring for $2 \mathrm{~min}$ at room temperature. Then $1 \mathrm{~mL}$ of the mixed solutions were poured onto a $10 \mathrm{~mm}$ plastic dish and placed in an incubator for $24 \mathrm{~h}$ at $37^{\circ} \mathrm{C}$.

\subsection{Characterization of hydrogels containing AgSD nanocrystals}




\subsubsection{Microstructure of hydrogels}

Hydrogels containing $\mathrm{AgSD} / \mathrm{NCT}$ were firstly frozen at $-80^{\circ} \mathrm{C}$ for $24 \mathrm{~h}$ and then lyophilized. The resulting dried gels were placed on an aluminum mount, coated with conductive gold. The morphology of AgSD/NCT immobilized in the gels was examined by a JSM-6510 scanning electron microscope (SEM, Japan) at an acceleration voltage of $15 \mathrm{kV}$. Hydrogels containing AgSD/Bulk were also observed as control.

\subsubsection{Drug release from hydrogels}

Hydrogels containing $1 \mathrm{mg} \mathrm{AgSD} / \mathrm{NCT}$ or $\mathrm{AgSD} / \mathrm{Bulk}$ (calculated as pure AgSD) were placed in $100 \mathrm{ml}$ of PBS in a flask (For comparison, gels containing AgSD/Bulk were prepared at genipin concentration of $1.65 \mathrm{mM}$ and $4.40 \mathrm{mM})$. Then the in vitro drug releases from hydrogels were evaluated by using a rotary shaker at the same condition as shown in section 2.3.2. At predetermined intervals, $50 \mu \mathrm{L}$ of medium was withdrawn and centrifuged at $14000 \mathrm{rpm}$ for $10 \mathrm{~min}$ at $4^{\circ} \mathrm{C}$ and equal blank medium was compensated immediately. Dissolution profiles of a commercial AgSD cream were also measured as control. AgSD content was measured by UHPLC method described above. Drug release was modelled by Peppas equation Eq. (1) as follow:

$$
\frac{M_{t}}{M_{\infty}}=k t^{n}
$$


Where $M_{t} / M_{\infty}$ was the fractional release at time $t, k$ was a rate constant and $n$ was the diffusional exponent [23].

\subsubsection{Stability of nanosuspensions}

The physical stability of AgSD/NCT in crosslinked CHI hydrogel was investigated after storage for 6 weeks at room temperature. Physical stability of AgSD/NCT dispersing in CHI slurry without crosslinking process was also evaluated as control. SEM particle morphology and drug release behavior were utilized for evaluation of storage stability of $\mathrm{AgSD} / \mathrm{NCT}$ in hydrogels. Particle size and dissolution velocity measurements were used for $\mathrm{AgSD} / \mathrm{NCT}$ in $\mathrm{CHI}$ slurry.

\subsection{Biocompatibility studies}

The biocompatibility of AgSD/NCT hydrogel was assessed by MTT assay. L929 fibroblasts were seeded in 96 -well plate at a density of $5 \times 10^{3}$ cells per well. Fibroblasts were cultivated for $24 \mathrm{~h}$ to obtain sub-confluence. Then fibroblasts were washed with saline solution and $100 \mu \mathrm{L}$ of samples containing $2 \mu \mathrm{g} / \mathrm{ml} \mathrm{AgSD}$ (diluted with the complete medium) were put in contact with the fibroblasts. The biocompatibility of blank gel was also evaluated and fresh complete medium was used as control. After $48 \mathrm{~h}$ incubation, the medium was removed, $20 \mu \mathrm{L}$ MTT solution $(5 \mathrm{mg} / \mathrm{ml})$ was added to each well. After $4 \mathrm{~h}$ exposure at $37^{\circ} \mathrm{C}$, the extractants were drained off gently and $150 \mu \mathrm{L}$ of DMSO was added to dissolve the formazan crystals. 
The dissolved solution was shaken continuously for $10 \mathrm{~min}$ by a shaker. The optical density of formazan solution was detected by an ELISA reader (Elx800) at $490 \mathrm{~nm}$. All assays were done with six parallel samples. The percentage of relative viability\% was calculated by Eq. (2), where $\mathrm{OD}_{\text {samples }}$ were absorbance of samples and $\mathrm{OD}_{\text {negative }}$ was of negative control.

$$
\text { Valiability } \%=\frac{O D_{\text {samples }}}{O D_{\text {negative }}} \times 100 \% \quad \text { Eq. (2) }
$$

2.7. In vitro antibacterial activity.

To evaluate the antimicrobial properties of AgSD loaded dressings, the following reference bacterial strains were used: Staphylococcus aureus (S. Aureus, ATCC 25923); Escherichia coli (E. Coli, ATCC 25922); Pseudomonas aeruginosa ( $P$. Aeruginosa, ATCC 27853). Before testing, bacteria were grown overnight in standard Luria Bertani (LB) medium at $37^{\circ} \mathrm{C}$.

AgSD bulk and nanocrystal suspensions and hydrogels containing AgSD nanocrystals were evaluated for bactericidal inhibition against S. aureus, E. coli, P. Aeruginosa. LB nutrient agar was used as culture media [24]. $100 \mu \mathrm{L}$ liquid culture medium with bacteria was spread on nutrient agar in a Petri dish to create a confluent lawn of bacterial growth. Then, $50 \mu \mathrm{L}$ of different AgSD samples were tailored into small pores with diameter of $3 \mathrm{~mm}$. Penicillin \& Streptomycin solution and blank hydrogels were used as positive and negative control, respectively. Commercial AgSD cream was also tested as a reference. The dishes were incubated for $24 \mathrm{~h}$ at $37^{\circ} \mathrm{C}$. The 
antibacterial activity was determined by measuring the diameter of inhibition zone $(\mathrm{mm})$. All experiments were performed in triplicate.

\subsection{Animal experiment}

\subsubsection{Animals}

Kunming male mice weighing between 20 25g were purchased from Experimental Animal Center of Academy of Military Medical Science (AMMS, Beijing, China). The animal use protocol in this study was reviewed and approved by the Experimental Animal center of AMMS. All animal work was conducted according to the national and international guidelines. At first, the animals were acclimatized at a temperature of $25 \pm 2^{\circ} \mathrm{C}$ and a relative humidity of $70 \pm 5 \%$ under natural light/dark conditions for 1 week and were fed with food and water ad libitum. Anesthesia was achieved with pentobarbital sodium (40 mg/kg, Biomedical, Beijing) prior to skin wounding.

\subsubsection{Wound model}

The acute burn wound model was performed as described previously, with modification $[25,26]$. A burn template was made from a $10 \mathrm{~cm} \times 10 \mathrm{~cm}$ plastic case with a opening window $(1 \mathrm{~cm} \times 1 \mathrm{~cm})$ in the middle of the bottom. The dorsum of each mouse was carefully shaved and laid on the middle of the burn template after anesthesia so that dorsal skin could expose through the opening window. Then the exposed dorsal skin of each skin was immersed in $75^{\circ} \mathrm{C}$ for $15 \mathrm{~s}$. This method was 
used to form a second-degree burn wound [25]. For the excision wound model, the dorsal hair of each mouse was shaved, and a piece of full-thickness skin $(1 \mathrm{~cm} \times 1 \mathrm{~cm})$ was excised with sterilized scissors [26].

In the excision wound and burn models, the mice were divided into 6 groups with 6 mice for each group: (1) untreated control (Untreated), (2) S. Aureus treatment only (Staph), (3) S. Aureus and AgSD cream treatment (Cream), (4) S. Aureus and blank gel treatment (Blank Gel), (5) S. Aureus and AgSD/Bulk hydrogel treatment (AgSD/Bulk Gel), (6) S. Aureus and AgSD/NCT hydrogel treatment (AgSD/NCT Gel). AgSD dosage in cream and hydrogels was equivalent to $1 \mathrm{mg}$. For all $S$. Aureus treatment groups, we applied $500 \mu \mathrm{L}$ of $S$. aureus $\left(1 \times 10^{8} \mathrm{CFU} / \mathrm{ml}\right)$ solution to a piece of clean gauze to produce a "bacterial dressing" that was immediately placed on the wounds. The wounds were then covered with the "bacterial dressings" and bandaged for $24 \mathrm{~h}$ to let the bacterial solution enter the wound area [27]. One day later, we changed the bacterial dressings to therapeutic dressings corresponding to the different groups. The wound dressings were changed daily until sacrifice. While changing the dressings, a close-up picture of each wound was taken. Wound area closure was measured by marking the area on a transparent graph sheet. The exact area of the unrecovered wound was remarked and calculated. The results of wound measurements on various days were expressed as percentage of wound closure calculated by Eq. (3) as follows:

$\%$ Wound closure $\left(\right.$ Area $_{0}-$ Area $\left._{\mathrm{t}}\right) /$ Area $_{0} \times 100 \%$ Eq. (3)

Where the Area $_{0}$ was the initiative wound area, and the Area $a_{t}$ represented the wound 
area on the measure day.

\subsubsection{Collection of tissue}

The mice were sacrificed by neck dislocation on the 21 st day after cutaneous injury. Wound skins were harvested and preserved in $10 \%$ neutral buffer formalin for histological and collagen deposition examination. Blood samples were also obtained and immediately transferred into tubes. After centrifugation at $8000 \mathrm{~g}$ for $10 \mathrm{~min}$, the serum samples were removed and stored at $-80^{\circ} \mathrm{C}$.

\subsection{Measure of cytokine levels}

Serum levels of vascular endothelial growth factor (VEGF)-A, interleukin (IL)-6, and transforming growth factor (TGF)- $\beta 1$ were measured with cytokine-specific ELISA kits (Dakewei, Shenzhen, China). Each measurement was performed according to the manufacturer's instructions. Cytokine levels in normal mice were used as negative control.

\subsection{Histopathological analysis}

\subsubsection{Hematoxylin and eosin (H\&E) staining}

The gross histopathological changes at the wound site were evaluated by H\&E staining. The skin tissues fixed in $10 \%$ neutral buffer formalin were embedded in 
paraffin. $5 \mu \mathrm{m}$ thick tissue sections were obtained and visualized under a light microscope after standard H\&E staining (E200, Nikon, Japan). Eight random fields from different sections in each group were evaluated and scoring was done by the method described by Greenhalgh et al. [28]. Briefly, scoring for each field was done from 1 to 12 as follows: i) 1 to 3 was given to none to minimal cell accumulation and granulation tissue; ii) 4 to 6 to thin immature granulation tissue that was dominated by inflammatory cells but has few fibroblasts, capillaries, or collagen deposition and minimal epithelial migration; iii) 7 to 9 to moderately thick granulation tissue which could range from being dominated by inflammatory cells to more fibroblasts and collagen deposition, extensive neovascularization, with epithelium ranging from minimal to moderate migration; and iv) 10 to 12 to thick, vascular granulation tissue dominated by fibroblasts and extensive collagen deposition, with epithelium partially to completely covering the wound.

\subsubsection{Picrosirius red staining for collagen}

Assessment of collagen in wound sections was done by staining with picrosirius red (Direct Red 80, Sigma Aldrich, USA) through a modified picrosirius procedure [29]. According to the birefringence pattern, collagen types were differentiated as (i) thick and denser collagen showing orange to red color and (ii) thinner collagen fibers showing green [30]. Stained sections were viewed under polarized light (DMI6000I, Leica, Germany) and quantification of collagen amounts was done in six random images from each group by using ImageJ software (Version 1.44). 


\subsection{Statistical analysis}

All data were expressed as means \pm standard deviation (SD). A one-way analysis of variance (ANOVA) was performed with SPSS software (Version 19.0). Significance was determined at $P<0.05$.

\section{Results}

\subsection{Particle size and morphology}

Particle sizes and PIs of AgSD nanocrystals during milling progress were shown in Supplementary Fig. 1. Routinely, the mean diameters of bulk AgSD crystalline drug materials were tens of micrometers and the size distributions were broad. With increasing milling velocity and times, the sizes gradually decreased to $0.290 \mu \mathrm{m}$ and the PIs settled around $0.240 \sim 0.250$.

The morphology of AgSD nanocrystals was observed using TEM (Supplementary Fig. 1B). After milling, AgSD bulk powders were transformed into flaky shape nanocrystals in the presence of the stabilizers. The particle size ranged from $200 \sim 400$ $\mathrm{nm}$, which was consistent with the data from Zetasizer.

\subsection{Dissolution of AgSD nanocrystals}


As shown in Supplementary Fig. 2, AgSD nanosuspensions exhibited a dramatic increase in drug dissolution rate compared with un-milled AgSD suspensions with the same stabilizer composition. The dissolution rate of nanoparticles was so fast that $60.9 \%$ had dissolved immediately after $1 \mathrm{~min}$ and almost all drugs had dissolved within 15 min. In contrast, less than $20 \%$ of $\mathrm{AgSD}$ was dissolved after $6 \mathrm{~h}$ from the bulk suspensions.

3.3. Microstructure of hydrogels containing AgSD nanocrystals

Figure 1.

As shown in Fig. 1, macroporous sponge-like scaffolds with pore size ranging from $100 \mu \mathrm{m}$ to $200 \mu \mathrm{m}$ could be obtained upon a lyophilization process. After further magnification well-dispersed AgSD nanocrystals could be found within the network, without obvious aggregations. AgSD nanocrystals maintained their initial particle size ranging from $200 \mathrm{~nm}$ to $400 \mathrm{~nm}$ inside the network. In hydrogels containing bulk AgSD powders, particle size of drugs was in range of tens of micrometers.

\subsection{Drug release}

After $48 \mathrm{~h}$, cumulative AgSD release was $92.49 \%, 73.70 \%, 66.84 \%$ and $55.23 \%$ for 
gels containing AgSD nanocrystals (Fig. 2A). It could be seen that the AgSD release rate from hydrogels decreased with increase of crosslinking density. On the other hand hydrogels containing AgSD nanocrystals released much faster than those containing AgSD bulk powders at a given crosslinking level (92.49\% vs 52.78\% for hydrogel with $1.65 \mathrm{mM}$ genipin; $55.23 \%$ vs $32.37 \%$ for hydrogel with $4.40 \mathrm{mM}$ genipin). It only reached $27.09 \%$ for commercial AgSD cream. The kinetic constant $k$ and diffusion constant $n$ of different gels were shown in Table 1. It could be seen that all release curves of different gels could be well fitted with the equation with coefficient $\mathrm{R}$ ranging from 0.958 to 0.978 .

Figure 2 .

Table 1 .

\subsection{Stability}

After 6-week storage, AgSD/NCT in $\mathrm{CHI}$ slurry showed visible caking and sedimentation on the bottom of the vial, but could be redispersed after moderate hand shaking. The mean particle size had increased to about $3.5 \mu \mathrm{m}$ and PI to 0.57 (Supplementary Fig. 3). When dissolution test as described in section 2.3.2 was carried out on $\mathrm{AgSD} / \mathrm{NCT}$ suspensions after 6-week storage, dissolution velocity markedly decreased (only about $60 \%$ had dissolved within 6h) compared to the 
freshly prepared AgSD/NCT suspensions. For the AgSD nanoparticles incorporated in hydrogels, however, SEM images indicated the original particle size of nanocrystals encapsulated in hydrogels could be maintained after 6-week storage (Fig. 1B). Besides, no obvious changes could be seen in the drug release behavior compared to the freshly prepared hydrogels (Fig. 2B).

\subsection{Biocompatibility study}

The relative viability (\%) of L929 fibroblasts after $48 \mathrm{~h}$ incubation was shown in Supplementary Fig. 4. At the same drug concentration, AgSD nanocrystals showed much lower viability (48.56\%) than bulk powder (68.28\%). However, when the AgSD nanocrystals were incorporated in hydrogels, their inhibition effects on cellular growth were significantly reduced. Correlation between cell viability and crosslinking level could be seen. AgSD nanocrystal hydrogels crosslinked with $1.65 \mathrm{mM}$ genipin showed the lowest viability of $70.14 \%$, when the genipin amount increased to $4.4 \mathrm{mM}$ cell viability could increase to almost $100 \%$. Blank gels (4.4mM genipin) showed higher cell viability compared with negative control but with no statistical significance $(P<0.05)$. Cell viability of AgSD cream was only $48.86 \%$, significantly lower than hydrogels containing the same drug concentration.

\subsection{In vitro antibacterial activity.}


Table 2 .

The inhibitory activity was determined against S. aureus, E. Coli and P. Aeruginosa respectively. Except for blank hydrogels, distinct inhibition zones were distinctly obtained for positive control and all AgSD formulations (Table 2). AgSD/NCT suspensions showed larger zone diameter on three bacteria than $\mathrm{AgSD} / \mathrm{Bulk}$ suspensions, although significant difference only could be seen in $S$. aureus and $P$. Aeruginosa groups. After incorporated in hydrogels, AgSD/NCT showed slightly decreased zone diameter $(P>0.05)$, but still had significantly larger zone diameter than positive control $(P<0.05)$. AgSD/NCT hydrogels were also significantly more effective than AgSD cream against $S$. aureus $(P<0.05)$.

\subsection{Wound contraction rate}

Figure 3.

During the healing process, wound contraction of the open wound area was observed, and results were shown in Fig. 3. In the acute burn model, the AgSD/NCT hydrogel had significantly smaller wound areas on day 14 and day 21 compared with other therapeutic groups $(P<0.05)$. AgSD/Bulk hydrogel resulted in significantly smaller wound areas compared with AgSD cream on day $21(P<0.05)$. Blank gel group also 
showed larger closure than AgSD cream but with no statistical significance $(P>0.05)$. The excision wound model showed similar results. The wound area in the AgSD/NCT hydrogel group was significantly smaller than the other 5 groups on day 7, day 14 and day $21(\mathrm{P}<0.05)$. AgSD/Bulk hydrogel also led to significantly smaller wound areas compared with AgSD cream on day $21(\mathrm{P}<0.05)$. However, blank gel group remained lager wound areas than cream group on day 21 in excision model, but having no significance $(P>0.05)$.

\subsection{Measure of cytokine levels}

Table 3.

The effects of different treatment on the cytokine levels were shown in Table 3. In the burn model, the serum VEGF-A level in three AgSD containing dressings were significantly higher than that in Staph group $(P<0.05)$. VEGF-A level in AgSD/NCT hydrogel group was higher than that in commercial cream group, but without any significance. TGF- $\beta 1$ level in AgSD/NCT hydrogel, AgSD/Bulk hydrogel, AgSD cream and blank gel groups was all significantly higher than that in Staph group $(P<0.05)$. Conversely, AgSD/NCT hydrogel, AgSD/Bulk hydrogel and AgSD cream significantly reduced IL-6 levels compared to Staph group. The down regulation of blank hydrogel on IL-6 level had no significance compared to that of Staph group. In the excision model, only AgSD/NCT hydrogel group had VEGF-A level significantly 
higher than Staph group, which was also markedly higher than Cream group. Only AgSD/NCT hydrogel had TGF- $\beta 1$ level markedly higher than Staph group. IL-6 level in AgSD/NCT hydrogel, AgSD/Bulk hydrogel and Cream groups was all significantly lower than that in Staph group $(P<0.05)$. And the reduction effect of both AgSD/NCT hydrogel and AgSD/Bulk hydrogel on IL-6 level was markedly stronger than that of AgSD cream.

\subsection{Histological analysis}

The H\&E stained sections of healing burn wounds of different groups on the 21 st day were presented in Fig. 4A. Scabs could still be observed in Untreated, Staph, Cream and blank gel groups. The wound sections of Untreated and Staph groups still showed presence of infiltration of inflammation cells with few capillaries and epithelial cells. In wound sections of Cream and blank gel groups, slight inflammatory reaction still existed along with thin epithelial layer and obvious proliferation of fibroblast. In wound sections of $\mathrm{AgSD} / \mathrm{Bulk}$ hydrogel and $\mathrm{AgSD} / \mathrm{NCT}$ hydrogel groups, fibroblasts had been predominant with almost no inflammatory reaction observed. Moreover, the AgSD/NCT hydrogel group was characterized by thick epithelial layer and many newborn blood vessels. Fig. 4B also showed the sections of excision wounds of different groups on the 21st day. Scabs were seen in Untreated and Staph groups, where inflammatory cell infiltration and gathering of macrophages were present. As was the case in burn model, the wound sections of Cream and blank gel groups 
showed thin epithelial layer and mild fibroblast proliferation with slight inflammatory reaction, and wound sections of $\mathrm{AgSD} / \mathrm{Bulk}$ hydrogel and $\mathrm{AgSD} / \mathrm{NCT}$ hydrogel groups presented thick epithelial layer and newborn blood vessels with flourishing fibroblasts. Histological scoring of blank gel group was significantly higher than that of Staph group in both wound models $(P<0.05)$. Histological scoring of Cream group was markedly higher than that of Untreated and Staph groups in both models $(P<0.05)$, but significantly lower than AgSD/NCT hydrogel group $(P<0.05)$.

\subsection{Collagen deposition analysis}

Table 4 .

The representative picrosirius red stained sections of burn and excision wounds in mice from different groups on the 21 st days were presented in Fig. 4A. In both wound models, the collagen fibers in the sections of Untreated and Staph groups mainly exhibited irregular greenish birefringence under polarized microscope. The proportion of green fibers in Cream and blank gel groups was lower than that of Untreated and Staph groups, but the orange fiber proportion was more. The orange fibers were predominant in $\mathrm{AgSD} / \mathrm{Bulk}$ hydrogel and $\mathrm{AgSD} / \mathrm{NCT}$ hydrogel groups. Quantitative analysis of collagen fibers (Table 4) showed that on the 21st day AgSD/NCT hydrogel group showed significantly higher total collagen fiber amount than AgSD cream group in both wound models $(P<0.05)$. In addition, the ratio of type I collagen 
concentration to type III collagen concentration in $\mathrm{AgSD} / \mathrm{NCT}$ hydrogel group was the highest among all the treated groups. While the Untreated and Staph groups had very low ratio of type I collagen amount to type III.

\section{Discussion}

In our present study, AgSD was comminuted to nanosized crystals by wet milling method. According to Ostwald-Freundlich equation and Noyes-Whitney equation [31, 32], it was generally thought that the huge surface area and saturation solubility resulting from particle radius in nanometer range leaded to the increased and accelerated dissolution velocity. This would be conductive to formation of high concentration gradient and then enhanced antibacterial activity on the wound surface. However, one issue should be paid more attentions despite the benefits generally brought by the increased dissolution rate. Extremely high dissolution rate of AgSD nanocrystals might result in too high local peak concentration, which might result in a short therapeutic action and also aggravate its cytotoxicity. When sustained drug release and reduction of cytotoxicity were expected, incorporation of drug nanocrystals in biodegradable hydrogels might be a promising approach.

The SEM images displayed that the crosslinking process had no effects on particle size and dispersion of nanocrystals inside the gels (Fig 1). Although the size dimension was kept in nanometer range, nanoparticles incorporated inside the gels no longer showed a burst dissolution behavior in PBS like the unencapsulated 
nanocrystals. Hydrogels of different crosslinking level exhibited a sustained and stable release within $48 \mathrm{~h}$ (Fig 2A). All the release curves of hydrogels fitted well with Peppas equation, which was generally considered to be applicable for drug release from swellable matrix. In Peppas equation, rate constant $k$ is a kinetic constant, a higher value of $k$ suggests a burst release of drug from matrix. Diffusional exponent $n$, calculated from the slope of the natural logarithmic values of fractional release as a function of time, provides an indication of the release mechanism and generally ranges from 0.5 to 1 . An $n$ value of 0.45 indicates Fickian diffusion whereas a value of 0.45-0.89 indicates anomalous transport, as there exists an influence of swelling and/or erosion [23]. The kinetic constant $k$ generally became lower with the increase of crosslinking extent of hydrogels (Table 1). The diffusional exponent $n$ values ranged from 0.549 to 0.626 , indicating that drug released from hydrogels through a swelling dependent mechanism, i.e drug release was limited by the hydration and swelling of the hydrogels in medium. To further verify the existence of correlation between crosslinking level and drug releasing behavior, we made a correlation study by using amount of crosslinking agent as $x$ axis and the product of $k$ and $n$ as $y$ axis (Fig. 2A). As a result a correlation coefficient $\mathrm{R}^{2}$ of 0.965 could be obtained, which also supported the swelling-dependent release pattern for hydrogels.

Our studies demonstrated that after 6-week storage particle growth and aggregation could be observed for nanocrystals dispersed in CHI slurry. Aggregation of nanocrystals mainly arises from the natural tendency of the nanoparticles to reduce the huge surface energy caused by the large interface between the solid and the 
medium [33]. Only coverage of stabilizers on the surface by non-covalent anchoring may not be enough to electrostatically or sterically stabilize the nanoparticles in suspension [34]. Moreover, nanoparticles highly dispersed in liquid tend to grow, which is called the Ostwald ripening phenomenon [35]. For the two reasons, particle size of nanocrystals tends to grow in liquid which finally leads to aggregation and caking. If the viscous $\mathrm{CHI}$ solution was further crosslinked, then nanocrystals were homogeneously and individually immobilized in situ and aggregation among nanoparticles could not occur any more. Meanwhile the Ostwald ripening phenomenon was also prevented due to the lack of free water for molecule solvation and migration inside the network [36]. Because hydrogels could not be tested with laser particle size analyzer, so distribution and agglomeration degree of AgSD nanocrystals were assessed by SEM images. Individual nanocrystals were still visible and well-distributed throughout the network after 6-week storage. The maintenance of uniform dispersion of AgSD nanocrystals in hydrogels was crucial to keep a stable and controlled drug release behavior.

Biocompatibility studies showed that AgSD was cytotoxic toward fibroblasts, which was in agreement with findings by other authors [9]. At the same drug concentration, AgSD nanocrystals showed less cell viability compared to AgSD bulk powders. It could be explained by the increased interaction between fibroblasts and particles caused by the high surface-to-volume ratio of nanocrystals and also the higher dissolved drug concentration resulted from the significant improved solubility. The encapsulation of AgSD nanocrystals in hydrogels significantly decreased the 
cytotoxic effect against fibroblasts. Generally, cytotoxic effect was associated with crosslinking level, hydrogel of higher crosslinking level showed lower cytotoxic effect. It might be because AgSD released from hydrogels of higher crosslinking level at a slower rate. Similar result had been reported by Lee et al, who found that cellular damage could be minimized by the sustained release of AgSD from collagen membrane [37]. The blank hydrogels displayed higher cell viability than negative control even at the highest crosslinking agent concentration, which was consistent with our previous report [22]. Interestingly, commercial AgSD cream at the same AgSD concentration showed higher cytotoxicity than AgSD bulk powders. Maybe the solubilization effect and cytomembrane permeability prompting effect of surfactants existing in the cream aggravated the cytotoxicity of $\mathrm{AgSD}[38]$.

Taking into consideration of low cytotoxicity effect and sustained release, we chose hydrogel with $4.40 \mathrm{mM}$ genipin for the antibacterial studies and wound healing experiments. AgSD/NCT suspensions showed larger inhibition zone size against three bacteria than AgSD/Bulk suspensions, which could also be explained by the enhanced interaction with bacteria and increased solubility of $\mathrm{AgSD} / \mathrm{NCT}$. When incorporated in hydrogel $\mathrm{AgSD} / \mathrm{NCT}$ displayed nearly the same zone size as unencapsulated nanocrystals. It indicated that extended release of AgSD from hydrogel contributed to lower cytotoxicity against fibroblast, but meanwhile it could ensure a sufficient in vitro antibacterial effect. This was probably because that the porous structure of the hydrogel contributed to penetration of bacterial liquid and a sufficient contact between bacteria and drugs release from the network. 
Acute cutaneous wound often inspires the regulation of various cytokines which play very important role in various physiological activities of wound healing, including inflammation reaction, reestablishment of blood vessels, fibroblast proliferation or collagen synthesis [39]. In the present study, VEGF-A, TGF- $\beta 1$, and IL-6 were chosen to represent three different aspects of the wound healing process after different treatments, i.e., angiogenesis, fibrogenesis, and inflammation [40-42]. VEGF-A is an important growth factor involved in vasculogenesis and angiogenesis, which subsequently support the high metabolic activity for cell proliferation and collagen synthesis [40]. Results in Table 3 showed that VEGF-A serum levels in AgSD/Bulk hydrogel and AgSD/NCT hydrogel groups were sharply up-regulated in both cutaneous wound models. Accordingly, new blood vessels could be clearly seen in H\&E stained images of the two groups (Fig. 4A). TGF- $\beta 1$ is a key cytokine that induces differentiation of fibroblasts into myofibroblasts which are in charge of collagen synthesis [42]. In the early phase of healing wounds, fibroblasts are recruited and induced in granulation tissue to synthesize collagen fibers rich in thin and loose type III collagen (green fibers after stained by picrosirius red) [43]. As the tissue recovery process advances, the granulation tissue is replaced by a more resistant tissue rich in type I collagen which is the main component of a mature scar (orange fibers after stained by picrosirius red) [43]. The high expression of TGF- $\beta 1$ in AgSD/Bulk hydrogel and AgSD/NCT hydrogel groups stimulated the fibroblasts to produce a dense collagen network. The great majority of collagen in wound sections of the two groups was type I fibers, indicating an almost complete wound healing. On 
the contrary, type III fibers accounted for a large proportion in Staph groups and Untreated groups of the two models, indicating that the wound healing was still in the initial phase. At 21 days post cutaneous injury the Staph groups (especially in burn model) still had high serum levels of IL-6, a kind of cytokine released by inflammatory cells in excessive inflammation. Accordingly, H\&E stained images of Staph groups presented severe inflammation reaction. However, almost no inflammation could be seen in AgSD/Bulk hydrogel and AgSD/NCT hydrogel groups, which showed significantly lower IL-6 level.

The best healing effects of $\mathrm{AgSD} / \mathrm{NCT}$ gels could be attributed to several reasons. Firstly, the moist local environment provided by hydrogels was conducive to wound healing [44]. Kant et al reported the similar results, they found that blank Pluronic F-127 gel gave rise to faster wound healing rate than normal saline due to its ability to provide a moisture environment to the wound surface [45]. Besides, CHI itself had been proved to have the ability to improve the ECM remodeling phase of wound healing [46]. Therefore, in our present study, blank gels leaded to faster wound closure, more collagen deposition but less inflammation reaction compared to Untreated and Staph groups. Secondly, antibacterial ability of AgSD could eliminate the destruction effects of bacteria on wound healing. Apart from this, AgSD also possesses ability to facilitate wound convergence, formation of granulation tissue and finally scar maturation [47]. After nanosization the antibacterial and wound convergence promoting abilities of AgSD were further enhanced compared to those of bulk powders due to the significant increase of interaction surface area. Thirdly, 
encapsulation of AgSD in hydrogels significantly reduced its cytotoxic effect against fibroblasts. All of these contributed to the best wound healing effects of AgSD/NCT hydrogels.

\section{Conclusion}

The present work mainly studied the advantage of genipin crosslinked CHI hydrogels as potential carriers for AgSD nanosystems. AgSD nanocrystals with a mean particle size of $289 \mathrm{~nm}$ were successfully prepared using wet milling technology. Although AgSD nanocrystals significantly enhanced the dissolution rate and in vitro antibacterial activity compared to bulk powders, the poor physical stability and severe cytotoxicity to fibroblasts were intractable disadvantages for clinical usage. To solve these issues AgSD nanocrystals were further encapsulated into CHI hydrogels. After immobilized in the hydrogels, AgSD nanocrystals displayed a very good physical stability and a swelling-dependent drug release pattern fitting well with Peppas equation. AgSD nanocrystals, immobilized in hydrogels, could significantly enhance the in vitro antibacterial effects due to the increased interaction area whilst their cytotoxicity had been significantly reduced. In vivo evaluation in excision and burn wound mice models showed that wounds treated with the AgSD/NCT containing hydrogels had the fastest healing rate, most collagen deposition and almost complete re-epithelialization compared to other dressings including AgSD cream, blank hydrogels and hydrogels containing AgSD/Bulk. Our findings proved that genipin 
crosslinked CHI hydrogel was a promising carrier for nanomedicines for local wound treatment.

\section{Conflict of interest}

None declared. 


\section{Acknowledgments}

This work was founded by the Newton Research Programme Foundation titled

“ Investigation of host tissue integration using closely integrated experimental and computational approaches approved by the Royal Academy of Engineering, the project number is NRCP/1415/54. 


\section{References}

[1] L.F. Wang, J.L. Li, W.H. Ma, J.Y. Li, Genet. Mol. Res. 13 (2014) 9727.

[2] T. Mayes, M.M. Gottschlich, L.E. James, C. Allgeier, J. Weitz, R.J. Kagan, J. Burn Care Res. 36 (2015) 92.

[3] D. Church, S. Elsayed, O. Reid, B. Winston, R. Lindsay, Clin. Microbiol. Rev. 19 (2006) 403-434.

[4] Lio PA, Kaye ET. Topical antibacterial agents. Infect Dis Clin North Am. 2009; 23 (4): 945 .

[5] T. Dai, Y.Y. Huang, S.K. Sharma, J.T. Hashmi, D.B. Kurup, M.R. Hamblin, Recent Pat. Antiinfect. Drug Discov. 5 (2010) 124.

[6] L. Jeong, M.H. Kim, J.Y. Jung, B.M. Min, W.H. Park, Int. J. Nanomedicine. 9 (2014) 5277 .

[7] A. Adhya, J. Bain, O. Ray, A. Hazra, S. Adhikari, G. Dutta, S. Ray, B.K. Majumdar, J. Basic Clin. Pharm. 6 (2014) 29.

[8] E. Dellera, M.C. Bonferoni, G. Sandri, S. Rossi, F. Ferrari, C. Del Fante, C. Perotti,

P. Grisoli, C. Caramella, Eur. J. Pharm. Biopharm. 88 (2014) 643.

[9] J. Rosen, A. Landriscina, A. Kutner, B.L. Adler, A.E. Krausz, J.D. Nosanchuk, A.J. Friedman, J. Invest. Dermatol. 135 (2015) 1459.

[10] L. Gao, G. Liu, J. Kang, M. Niu, Z. Wang, H. Wang, J. Ma, X. Wang, Colloids Surf. B Biointerfaces. 111 (2013) 277. 
[11] L. Gao, G. Liu, X. Wang, F. Liu, Y. Xu, J. Ma, Int. J. Pharm. 404 (2011) 231.

[12] M. Han, X. Yu, Y. Guo, Y. Wang, H. Kuang, X. Wang, Colloids Surf. B Biointerfaces. 116 (2014) 114.

[13] S. Agnihotri, G. Bajaj, S. Mukherji, S. Mukherji, Nanoscale.; 7 (2015) 7415.

[14] X. Li, J.J. Lenhart, H.W. Walker, Langmuir. 28 (2012) 1095.

[15] S. Nagarajan, K. Arumugam Kuppusamy, India. J. Nanobiotechnology. 11 (2013) 39.

[16] Y. Wang, Y. Zheng, L. Zhang, Q. Wang, D. Zhang, J. Control. Release. 172 (2013) 1126.

[17] Y. Zhao, X. Zhang, Y. Wang, Z. Wu, J. An, Z. Lu, L. Mei, C. Li, Carbohydr. Polym. 105 (2014) 63.

[18] K.C. Broussard, J.G. Powers, Am. J. Clin. Dermatol. 14 (2013) 449.

[19] C. Martin, W.L. Low, M.C. Amin, I. Radecka, P. Raj, K. Kenward, Pharm. Pat. Anal. 2 (2013) 341.

[20] A. Sood, M.S. Granick, N.L. Tomaselli, Adv. Wound Care. 3 (2014) 511.

[21] K. Lay-Flurrie, Prof. Nurse. 19 (2004) 269.

[22] L. Gao, H. Gan, Z. Meng, R. Gu, Z. Wu, L. Zhang, X. Zhu, W. Sun, J. Li, Y. Zheng, G. Dou, Colloids Surf. B Biointerfaces. 117 (2014) 398.

[23] Q. Zhou, L. Zhong, X. Wei, W. Dou, G. Chou, Z. Wang, Int. J. Pharm. 454 (2013) 125.

[24] N. Tanideh, M.H. Haddadi, M.H. Rokni-Hosseini, M. Hossienzadeh, D. Mehrabani, K. Sayehmiri, O. Koohi-Hossienabadi, World J. Plast. Surg. 4 (2015) 16. 
[25] J.C. Wu, L.F. Rose, R.J. Christy, K.P. Leung, R.K. Chan, Adv. Wound Care. 4 (2015) 83 .

[26] MA. Abu-al-Basal, Pak. J. Biol. Sci. (2012) 306.

[27] C.Y. Chu, F.C. Peng, Y.F. Chiu, H.C. Lee, C.W. Chen, J.C. Wei, J.J. Lin, PLoS One. 7 (2012) e38360.

[28] D.G. Greenhalgh, K.H. Sprugel, M.J. Murray, R. Ross, Am. J. Pathol. 136 (1990) 1235.

[29] D. Dayan, Y. Hiss, A. Hirshberg, J.J. Bubis, M. Wolman, Histochemistry 93 (1989) 27.

[30] D. Rizzoni, E. Porteri, C. Ciuceis, I. Sleiman, L. Rodella, R. Rezzani, S. Paiardi, R. Bianchi, G. Ruggeri, G.E. Boari, M.L. Muiesan, M. Salvetti, F. Zani, M. Miclini, E.A. Rosei, Hypertension 45 (2005) 659.

[31] B.H.L. Böhm, R.H. Müller, Pharm. Sci. Technol. Today 2 (1999) 336.

[32] A. Dokoumetzidis, V. Papadopoulou, P. Macheras, Pharm. Res. 23 (2006) 256.

[33] Y. Wang, Y. Zheng, L. Zhang, Q. Wang, D. Zhang, J. Control. Release. 172 (2013) 1126.

[34] X. Zhai, J. Lademann, C.M. Keck, R.H. Müller, Eur. J. Pharm. Biopharm. 88 (2014) 85 .

[35] S. Verma, S. Kumar, R. Gokhale, D.J. Burgess, Int. J. Pharm. 406 (2011) 145.

[36] B. Obradovic, J. Stojkovska, Z. Jovanovic, V. Miskovic-Stankovic, J. Mater. Sci. Mater. Med. 23 (2012) 99.

[37] J.E. Lee, J.C. Park, K.H. Lee, S.H. Oh, J.G. Kim, H. Suh, Artif. Organs. 26 (2002) 
636.

[38] D.R. Nogueira, M. Mitjans, M.C. Morán, L. Pérez, M.P. Vinardell, Amino. Acids. 43 (2012) 1203.

[39] T. Muthukumar, K. Anbarasu, D. Prakash, T.P. Sastry, Colloids Surf. B Biointerfaces. 121 (2014) 178.

[40] L. Zhang, Z. Wu, H. Qin, W. Chen, G. Zhang, Wounds. 26 (2014) 293.

[41] L.R. Luckett, R.M. Gallucci, Br. J. Dermatol. 156 (2007) 1163.

[42] L.W. Reneker, A. Bloch, L. Xie, P.A. Overbeek, J.D. Ash, Brain Res. Bull. 81 (2010) 287.

[43] M. Ganeshkumar, T. Ponrasu, R. Krithika, K. Iyappan, V.S. Gayathri, L. Suguna, J. Ethnopharmacol. 142 (2012) 14.

[44] H.C. Korting, C. Schöllmann, R.J. White, J. Eur. Acad. Dermatol. Venereol. 25 (2011) 130 .

[45] V. Kant, A. Gopal, N.N. Pathak, P. Kumar, S.K. Tandan, D. Kumar, Int. Immunopharmacol. 20 (2014) 322.

[46] H. Ueno, F. Nakamura, M. Murakami, M. Okumura, T. Kadosawa, T. Fujinag, Biomaterials. 22 (2001) 2125.

[47] P. Suman, H. Ramachandran, S. Sahakian, K.Z. Gill, B.A. Horst, S.M. Modak, M.A. Hardy, Ann. N. Y. Acad. Sci. 1270 (2012) 28. 


\section{Figure captions}

Fig. 1. (A) Comparison of genipin crosslinked CHI hydrogels containing AgSD nanocrystals and AgSD bulk powders. (B) SEM image of AgSD/NCT after 6-week storage.

Fig. 2. (A) AgSD release profiles from CHI hydrogels of different crosslinking level and commercial cream. Values are expressed as means $\pm \operatorname{SD}(n=4)$. The insert graph showed the correlation between genipin level in hydrogels and product of $k$ and $n$. (B) Drug release profile of freshly prepared AgSD/NCT hydrogels and AgSD/NCT hydrogels after 6-week storage.

Fig. 3. Photographs and wound closure $\%$ of wounds at 7, 14 and 21 days. (A) Photographs of wounds treated by different dressings at $0,7,14$ and 21 days post burn or excision injury; (B) Wound closure\% of wounds at 7, 14 and 21 days post burn injury and excision injury $\left(\mathrm{n}=6,{ }^{*} P<0.05\right.$, vs Staph group; ${ }^{\triangle} P<0.05$, vs AgSD cream group; ${ }^{\#} P<0.05$, vs AgSD/Bulk hydrogel group).

Fig. 4. (A): H\&E and Sirius red stained images of burn or excision mice (a: Untreated group; b: Staph group; c: Cream group; d: Blank gel group; e: AgSD/Bulk hydrogel group; f: AgSD/NCT hydrogel group; BV: blood vessels; E: epithelial layer; F: fibroblasts; I: inflammation cells; M: macrophages). In the H\&E images, the scab still could be seen in Untreated and Staph groups, as well as inflammatory cell infiltration and macrophage gathering. Fibroblasts, collagen deposition and epithelial layer were hardly observed in the two groups. Slight inflammatory cell infiltration still could be 
seen in granulation tissues of Cream and blank gel groups, but also the moderate collagen deposition, fibroblast proliferation and thin epithelial layer; Fibroblasts were dominant in $\mathrm{AgSD} / \mathrm{Bulk}$ and $\mathrm{AgSD} / \mathrm{NCT}$ hydrogel groups with dense collagen fibers, thick epithelial layer and almost no inflammatory cell. (B) The average H\&E scoring of different wound sections (n=6, ${ }^{*} P<0.05$, vs Staph group; ${ }^{\wedge} P<0.05$, vs AgSD cream group; ${ }^{\#} P<0.05$, vs AgSD/Bulk hydrogel group). 


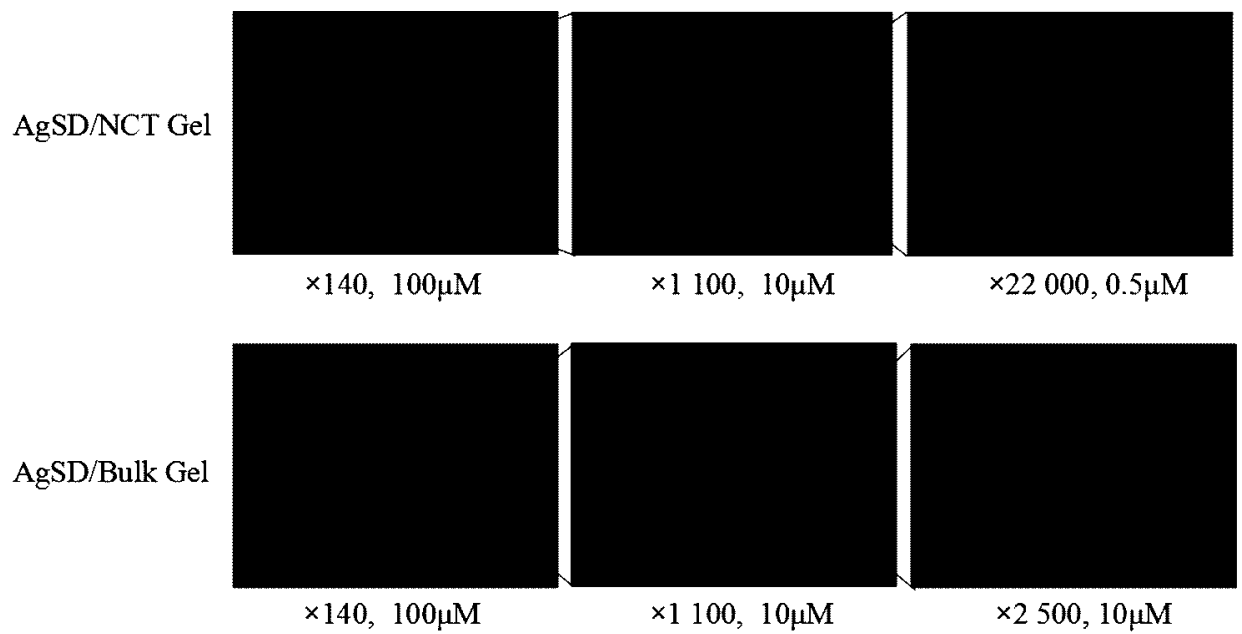

A

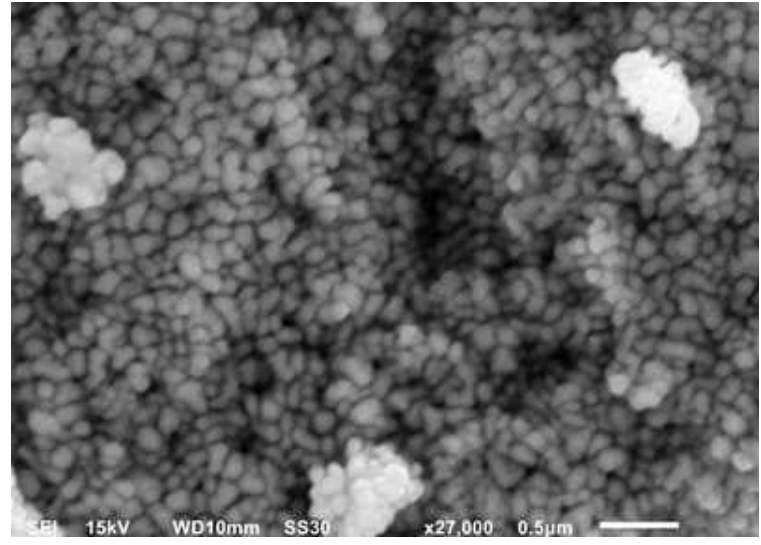

B

Fig. 1. 


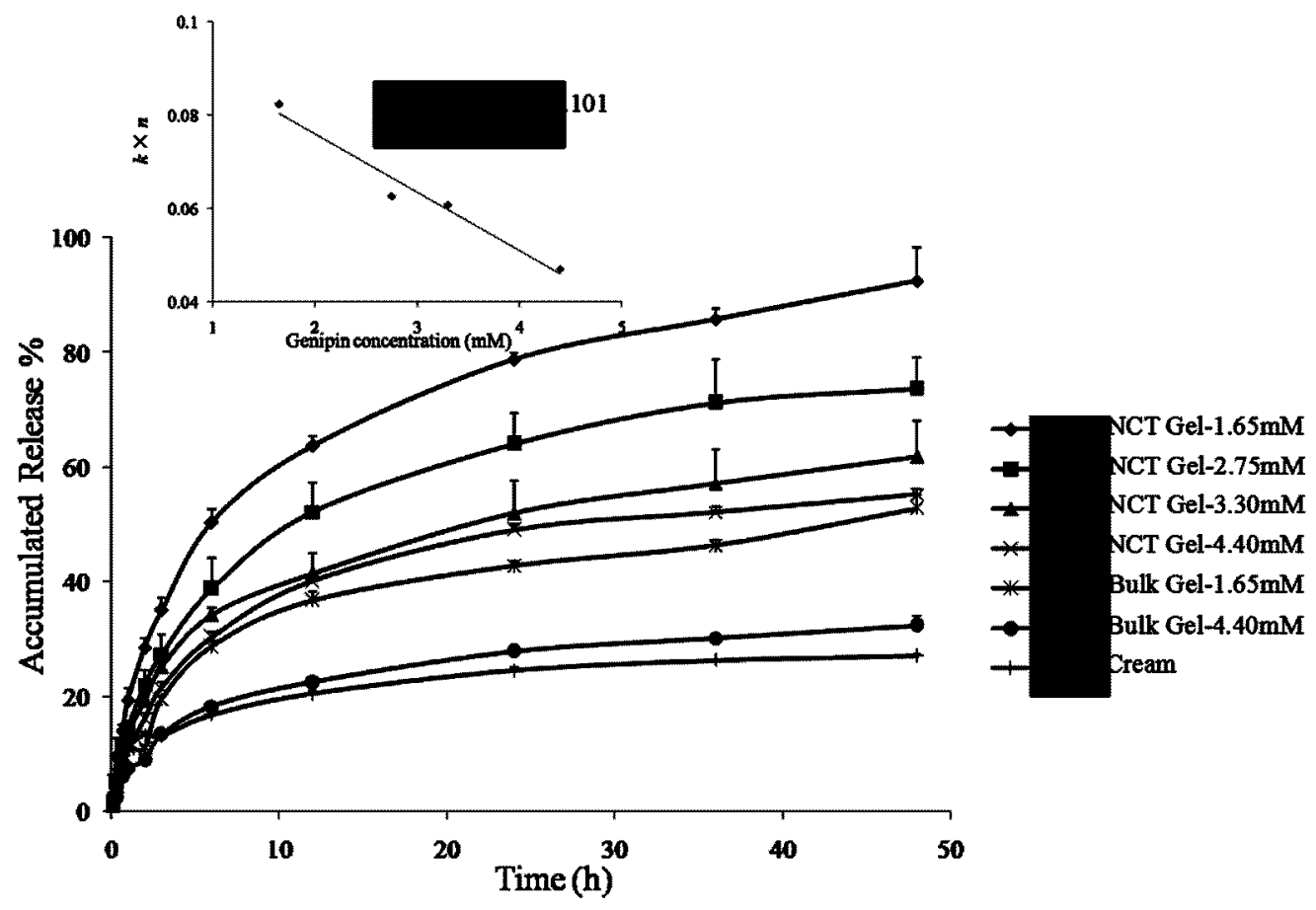

A

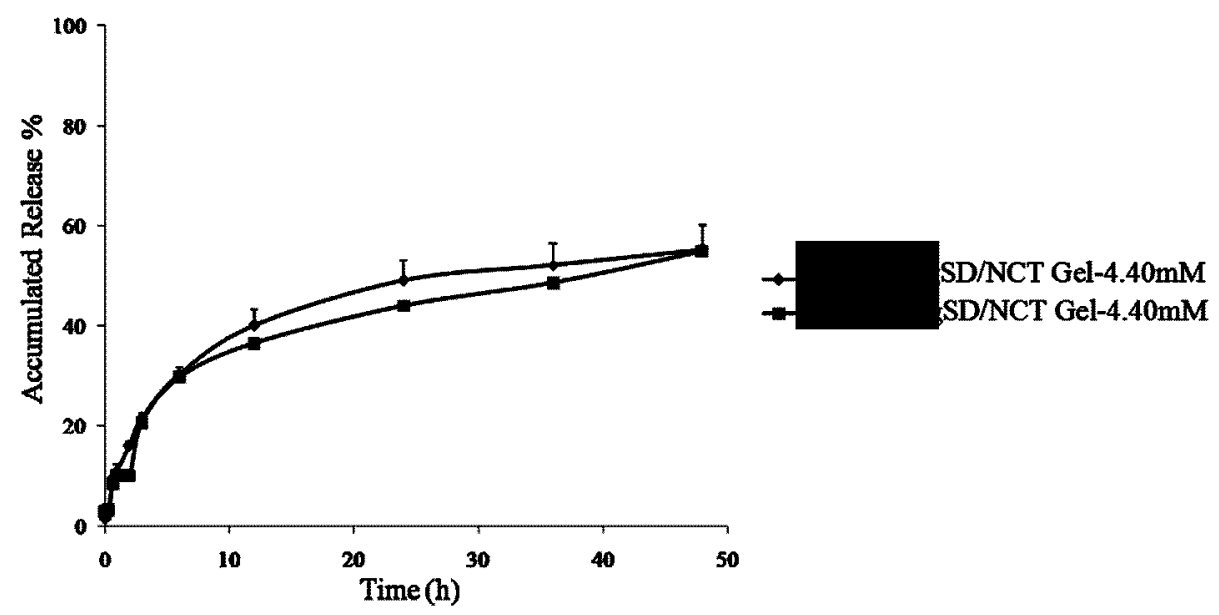

B

Fig. 2 . 


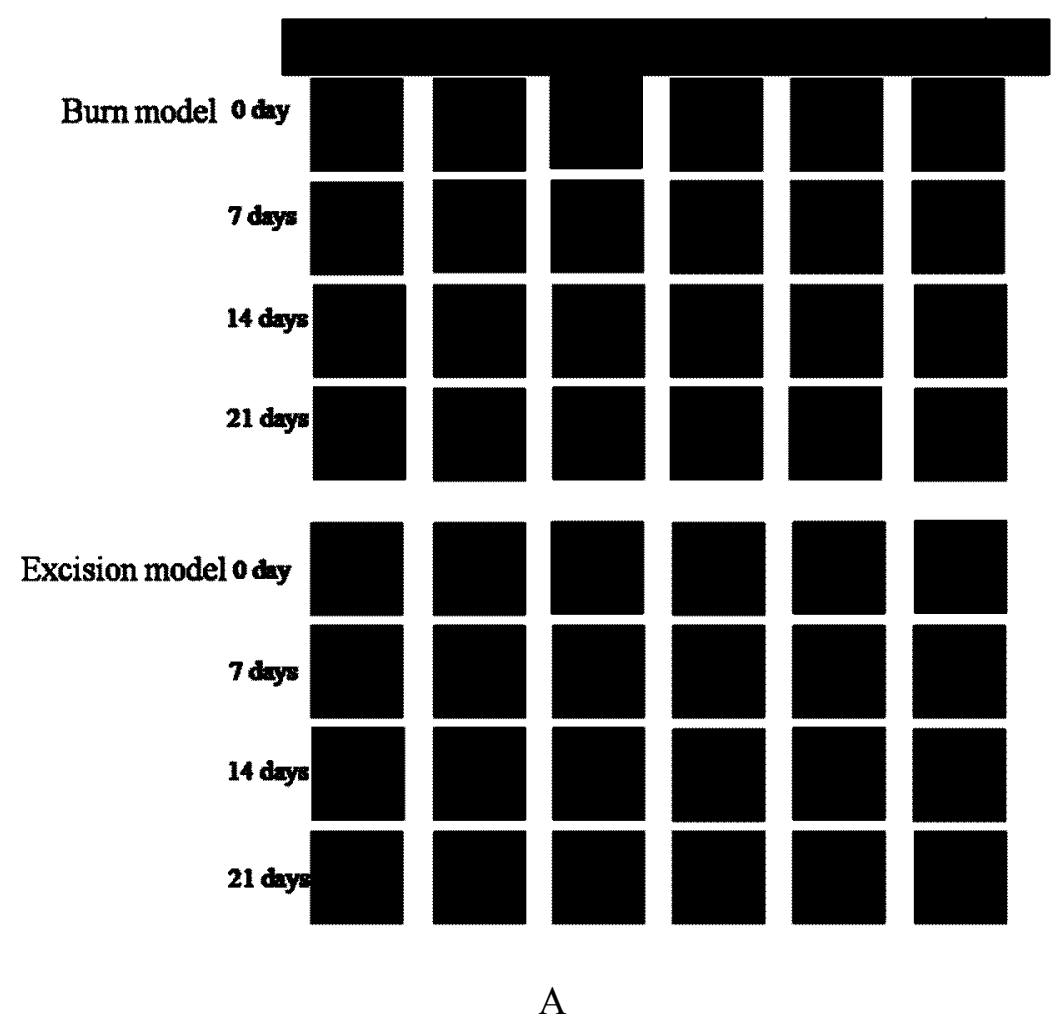

Burn model

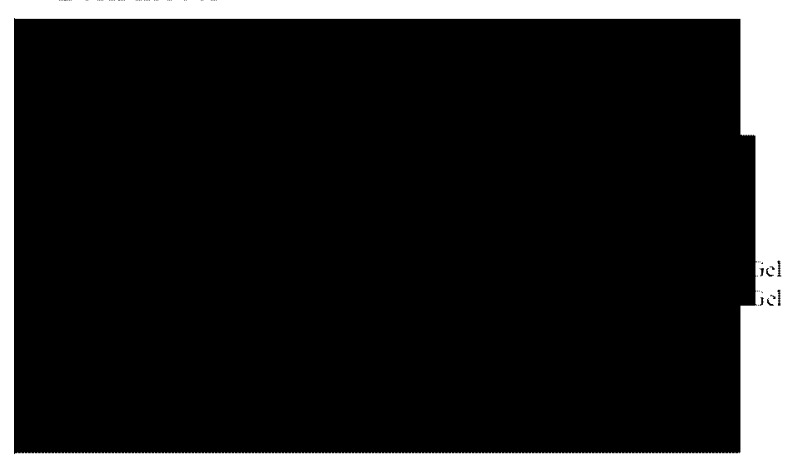

Excision model

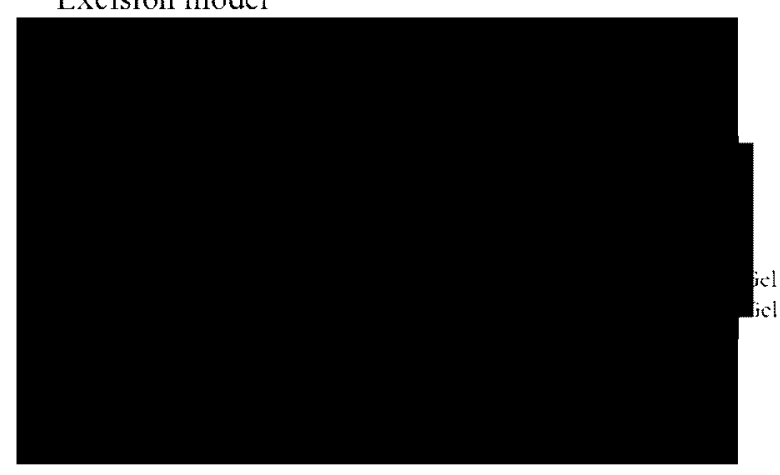

B

Fig. 3 . 


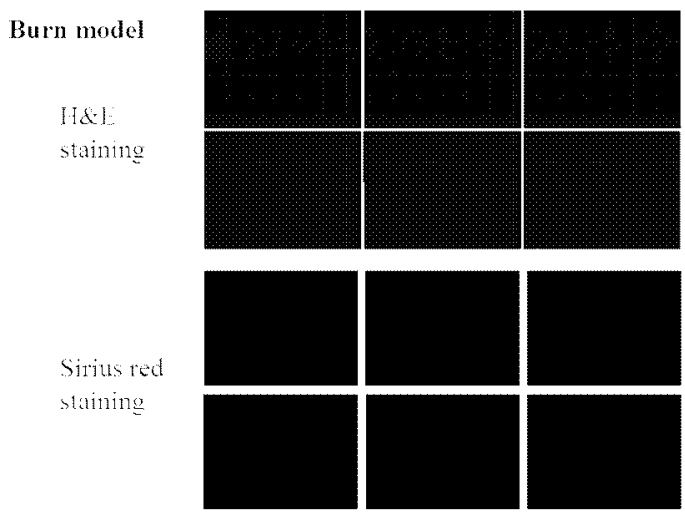

Excision model

H\&

staining

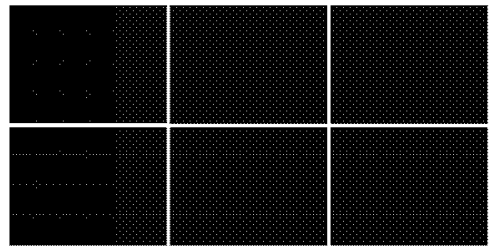

Sirus red

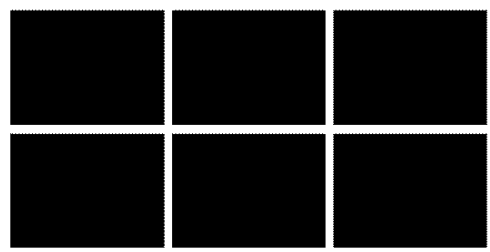

A

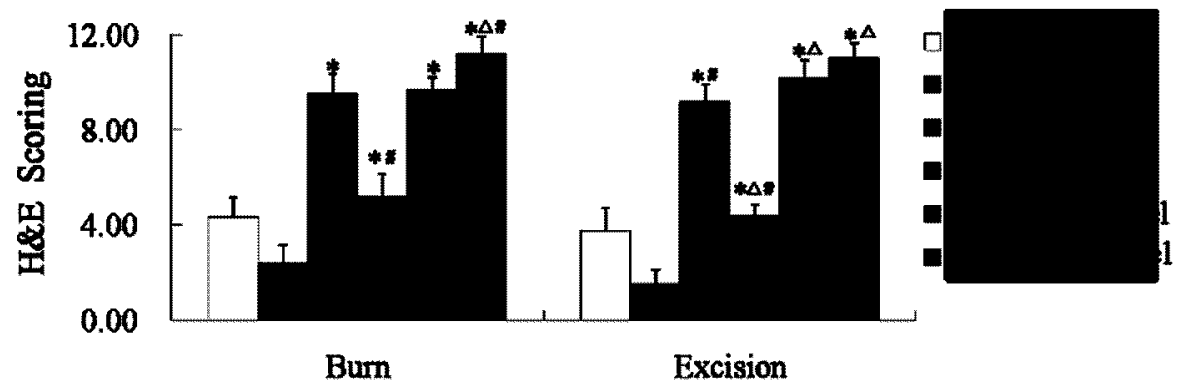

B

Fig. 4. 
Table 1. Parameters of drug release profiles of different hydrogels

\begin{tabular}{cccc} 
Sample & $k$ & $n$ & $\mathrm{R}$ \\
\hline AgSD/NCT Gel-1.65mM & 0.15 & 0.549 & 0.969 \\
AgSD/NCT Gel-2.75mM & 0.10 & 0.626 & 0.958 \\
AgSD/NCT Gel-3.30mM & 0.11 & 0.552 & 0.978 \\
AgSD/NCT Gel-4.40mM & 0.08 & 0.587 & 0.972 \\
AgSD/Bulk gel-1.65mM & 0.084 & 0.538 & 0.975 \\
AgSD/Bulk gel-4.40mM & 0.055 & 0.536 & 0.970 \\
\hline
\end{tabular}


Table 2. Zone diameter of inhibition values (mm) of different AgSD formulations against $S$. aureus, E. coli and $P$. aeruginosa (means $\pm \mathrm{SD}, \mathrm{n}=3$ )

\begin{tabular}{llll}
\hline & S. aureus & E. coli & P. aeruginosa \\
\hline Positive & $14 \pm 0.9$ & $13 \pm 1.9$ & $10 \pm 0.6^{a}$ \\
AgSD cream & $15 \pm 1.5$ & $12 \pm 0.6$ & $13 \pm 0.9^{\mathrm{a}, \mathrm{b}}$ \\
Blank gels & $5 \pm 0.5^{\mathrm{a}, \mathrm{b}, \mathrm{c}}$ & $5 \pm 0.5^{\mathrm{a}, \mathrm{b}, \mathrm{c}}$ & $4 \pm 0.2^{\mathrm{a}, \mathrm{b}, \mathrm{c}}$ \\
AgSD/NCT gels & $17 \pm 0.8^{\mathrm{b}}$ & $13 \pm 1.1$ & $15 \pm 0.3^{\mathrm{a}, \mathrm{b}}$ \\
AgSD/Bulk & $14 \pm 1.5$ & $11 \pm 1.4$ & $12 \pm 0.9^{\mathrm{c}}$ \\
AgSD/NCT & $18 \pm 0.8^{\mathrm{a}, \mathrm{b}, \mathrm{c}}$ & $14 \pm 2.0$ & $15 \pm 0.4^{\mathrm{a}, \mathrm{b}}$ \\
\hline
\end{tabular}

a $, P<0.05, \mathrm{vs}$ AgSD/Bulk; ${ }^{\mathrm{b}}, P<0.05$,vs Positive; ${ }^{\mathrm{c}}, P<0.05, \mathrm{vs}$ AgSD cream 
Table 3. Average serum concentration of VEGF-A, TGF- $\beta 1$ and IL-6 in burn and excision mice (means $\pm \mathrm{SD}, \mathrm{n}=6$ )

\begin{tabular}{|c|c|c|c|c|c|c|c|}
\hline & Cytokines & Untreated & Staph & Cream & Gel & AgSD/Bulk & $\mathrm{AgSD} / \mathrm{NCT}$ \\
\hline \multirow{3}{*}{ Burn model } & $\begin{array}{l}\text { VEGF-A } \\
(\mathrm{pg} / \mathrm{ml})\end{array}$ & $\begin{array}{l}113.8 \pm \\
7.8\end{array}$ & $\begin{array}{l}128.5 \pm \\
26.8\end{array}$ & $\begin{array}{l}247.0 \pm \\
83.1^{\mathrm{a}}\end{array}$ & $\begin{array}{l}133.7 \pm \\
27.3^{\mathrm{c}}\end{array}$ & $\begin{array}{l}199.5 \pm \\
20.4^{\mathrm{a}}\end{array}$ & $\begin{array}{l}303.7 \pm \\
34.3^{\mathrm{a}, \mathrm{c}}\end{array}$ \\
\hline & $\begin{array}{l}\text { TGF- } \beta 1 \\
(\mathrm{ng} / \mathrm{ml})\end{array}$ & $9.9 \pm 2.1^{\mathrm{a}}$ & $5.6 \pm 3.9$ & $10.7 \pm 2.2^{\mathrm{a}}$ & $\begin{array}{l}10.5 \pm \\
1.9^{\mathrm{a}}\end{array}$ & $\begin{array}{l}11.4 \pm \\
1.3^{\mathrm{a}}\end{array}$ & $12.3 \pm 5.2^{\mathrm{a}}$ \\
\hline & $\begin{array}{l}\text { IL-6 } \\
(\mathrm{pg} / \mathrm{ml})\end{array}$ & $400.4 \pm 175$ & $7522.3 \pm 47.0$ & $197.0 \pm 56.6^{\mathrm{a}}$ & $\begin{array}{l}394.7 \pm \\
111.0\end{array}$ & $274.9 \pm 35.5^{\mathrm{a}}$ & $217.3 \pm 38.9^{\mathrm{a}}$ \\
\hline \multirow{3}{*}{$\begin{array}{c}\text { Excision } \\
\text { model }\end{array}$} & $\begin{array}{l}\text { VEGF-A } \\
(\mathrm{pg} / \mathrm{ml})\end{array}$ & $188.3 \pm 42.7$ & $187.5 \pm 57.0$ & $231.9 \pm 52.6$ & $190.6 \pm 93.0$ & $231.0 \pm 76.1$ & $\begin{array}{l}435.1 \pm \\
99.0^{\mathrm{a}, \mathrm{b}, \mathrm{c}}\end{array}$ \\
\hline & $\begin{array}{l}\text { TGF- } \beta 1 \\
(\mathrm{ng} / \mathrm{ml})\end{array}$ & $11.3 \pm 2.0^{\mathrm{a}}$ & $7.7 \pm 3.1$ & $10.9 \pm 0.5$ & $10.7 \pm 2.1$ & $10.4 \pm 4.0$ & $13.8 \pm 3.8^{\mathrm{a}}$ \\
\hline & $\begin{array}{l}\text { IL-6 } \\
(\mathrm{pg} / \mathrm{ml})\end{array}$ & $307.5 \pm 80.6$ & $394.2 \pm 28.4$ & $\begin{array}{l}271.7 \pm \\
12.2^{\mathrm{a}, \mathrm{c}}\end{array}$ & $292.3 \pm 72.1$ & $\begin{array}{l}237.6 \pm \\
22.6^{\mathrm{a}, \mathrm{b}}\end{array}$ & $87.8 \pm 35.6^{\mathrm{a}, \mathrm{b}, \mathrm{c}}$ \\
\hline
\end{tabular}

${ }^{\mathrm{a}} P<0.05$, vs Staph group; ${ }^{\mathrm{b}} P<0.05$, vs AgSD cream group; ${ }^{\mathrm{c}} P<0.05$, vs AgSD/Bulk hydrogel group. 
Table 4. Average values of type I and type III collagen fiber concentration (\%) and the ratio of type I to type III (means $\pm \mathrm{SD}, \mathrm{n}=6$ )

\begin{tabular}{lllllll}
\hline & Untreated & Staph & Cream & Gel & AgSD/Bulk & AgSD/NCT \\
\hline Burn model & & & & & & \\
Collagen type I & $1.25 \pm 0.86$ & $0.58 \pm 0.28$ & $4.51 \pm 1.48^{\mathrm{a}, \mathrm{b}, \mathrm{c}}$ & $3.91 \pm 1.20^{\mathrm{a}, \mathrm{c}}$ & $7.31 \pm 2.05^{\mathrm{a}, \mathrm{b}}$ & $10.86 \pm 1.41^{\mathrm{a}, \mathrm{b}, \mathrm{c}}$ \\
Collagen type III & $10.33 \pm 3.29$ & $9.35 \pm 2.06$ & $8.85 \pm 1.75$ & $9.44 \pm 2.44$ & $7.43 \pm 0.98$ & $4.98 \pm 1.65^{\mathrm{a}, \mathrm{b}}$ \\
Collagen I+III & $11.58 \pm 3.46$ & $9.93 \pm 1.87$ & $13.36 \pm 2.24^{\mathrm{a}}$ & $13.34 \pm 1.74^{\mathrm{a}}$ & $14.74 \pm 2.11^{\mathrm{a}}$ & $15.84 \pm 1.45^{\mathrm{a}, \mathrm{b}}$ \\
Ratio of I to III & $0.13 \pm 0.11$ & $0.07 \pm 0.04$ & $0.53 \pm 0.19^{\mathrm{a}}$ & $0.47 \pm 0.27$ & $1.00 \pm 0.36^{\mathrm{a}}$ & $2.53 \pm 1.41^{\mathrm{a}}$ \\
Excision model & & & & & & \\
Collagen type I & $2.54 \pm 2.12$ & $1.38 \pm 0.33$ & $9.45 \pm 3.13^{\mathrm{a}}$ & $7.02 \pm 1.60^{\mathrm{a}}$ & $12.44 \pm 3.89^{\mathrm{a}}$ & $17.88 \pm 5.20^{\mathrm{a}, \mathrm{b}}$ \\
Collagen type III & $9.78 \pm 2.83$ & $9.00 \pm 1.94$ & $5.92 \pm 2.92^{\mathrm{a}}$ & $7.28 \pm 2.00$ & $4.82 \pm 2.03^{\mathrm{a}}$ & $2.95 \pm 2.31^{\mathrm{a}, \mathrm{b}}$ \\
Collagen I+III & $12.29 \pm 1.97$ & $10.37 \pm 1.74$ & $15.37 \pm 3.08^{\mathrm{a}}$ & $14.30 \pm 2.44^{\mathrm{a}}$ & $17.26 \pm 3.40^{\mathrm{a}}$ & $20.82 \pm 3.38^{\mathrm{a}, \mathrm{b}, \mathrm{c}}$ \\
Ratio of I to III & $0.34 \pm 0.38$ & $0.16 \pm 0.05$ & $2.18 \pm 1.51$ & $1.02 \pm 0.32$ & $4.51 \pm 6.12$ & $9.49 \pm 7.04^{\mathrm{a}, \mathrm{b}}$ \\
\hline
\end{tabular}

${ }^{\mathrm{a}} P<0.05$, vs Staph group; ${ }^{\mathrm{b}} P<0.05$, vs AgSD cream group; ${ }^{\mathrm{c}} P<0.05$, vs AgSD/Bulk hydrogel group. 